\title{
Pharmacokinetic characterization of the population with hemophilia A in Spain, using an online medical application based on a published population model and a Bayesian algorithm.
}

\author{
Álvarez-Román $\mathrm{MT}^{1 *}$, Berrueco-Moreno $\mathrm{R}^{2}$, Bonanad-Boix $\mathrm{S}^{3}$, Canaro-Hirnyk $\mathrm{M}^{4}$, , García-Candel $\mathrm{F}^{5}$,López-Fernández \\ $\mathrm{MF}^{6}$, Mingot-Castellano $\mathrm{ME}^{7}$, Moretó-Quintana $\mathrm{A}^{8}$, Núñez-Vázquez $\mathrm{R}^{9}$, Paloma-Mora $\mathrm{MJ}^{10}$, Palomo-Bravo $\mathrm{A}^{11}$, \\ Rodríguez-López $\mathrm{M}^{12}$, Santamaría Ortíz $\mathrm{A}^{13}$ Soto-Ortega $\mathrm{I}^{14}$, Jiménez-Yuste $\mathrm{V}^{1}$, on behalf of the Spanish Society of \\ Thrombosis and Haemostasis prophylaxis group₹.
}

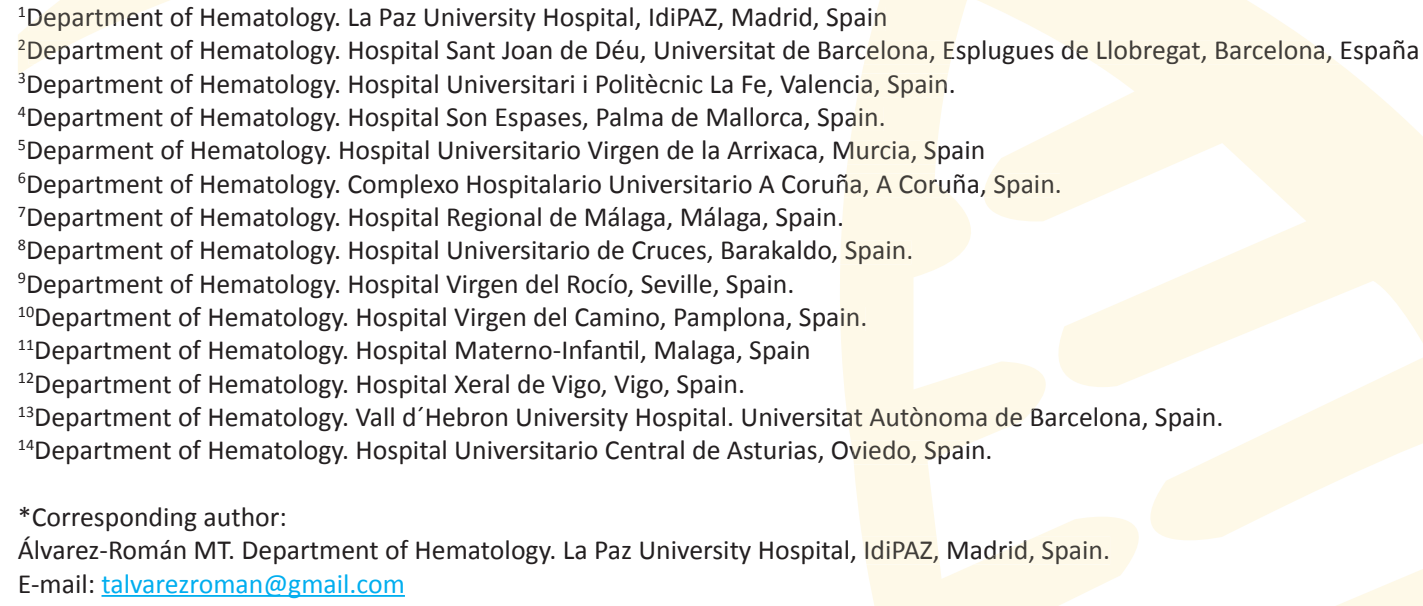

Objective: The main objective of this prospective observational study is to describe the pharmacokinetic profile of patients with hemophilia A in prophylaxis with Advate ${ }^{\circledR}$ in Spain using myPKFiT ${ }^{\circledR}$. Secondary objectives are (1) the characterization of the educational role of pharmacokinetics, (2) identifying whether there are any changes in attitude among the patients after receiving the educational information on pharmacokinetics and (3) evaluating the role of pharmacokinetics and other individual factors in patients with hemophilia A undergoing prophylactic treatment in Spain.

Methods: This is an observational, prospective (EPA-SP) and multicenter study. It will be conducted in 14 centers in Spain and will include patients with diagnosis of hemophilia A undergoing prophylactic treatment with Advate ${ }^{\circledR}$ and will use myPKFiT ${ }^{\circledR}$. The study will also include a cohort of patients who are already using myPKFiT ${ }^{\circledR}$ for the $\mathrm{PK}^{-g u i d e d}$ prophylaxis dose adjustment.

Discussion: Several studies have demonstrated the superiority of prophylactic treatment versus on-demand treatment. In order to make this prophylaxis more effective in preventing bleeding, it is necessary to adjust the treatment to several factors such as the physical activity that the patient performs, joint condition and the pharmacokinetic parameters of the infused factor VIII (clearance, life mean, area under the curve). Understanding the pharmacokinetic will help us personalize the treatment in our patients.

Trial Registration: ClinicalTrials.gov: NCT03006965.

Keywords: Pharmacokinetic, hemophilia, online medical aplication, Bayesian algorithm.

Received April 20, 2017 Accepted August 25, 2017 Published October 21, 2017.

Copyright: (c) 2017 Author. This is an open-access article distributed under the terms of the Creative Commons Attribution License, which permits unrestricted use, distribution, and reproduction in any medium, provided the original author and source are credited. Editor: Alberto Borobia

Cite as: Álvarez-Román MT, Berrueco-Moreno R, Bonanad-Boix S, Canaro-Hirnyk M, Santamaría Ortíz A, García-Candel F, et al. Pharmacokinetic characterization of the population with hemophilia A in Spain, using an online medical application based on a published population model and a Bayesian algorithm. IBJ Clin Pharmacol 2017 1(1):e0009.

Funding: This work is supported by an unrestricted grant by Shire to Spanish Society of Thrombosis and Haemostasis. Competing Interests: The authors declare no conflicts of interest. 


\section{BACKGROUND}

Hemophilia A is an inherited disorder characterized by coagulation factor VIII (FVIII) deficiency. The absence of FVIII leads to spontaneous hemorrhagic episodes (mainly in joints and muscles and less frequently in soft tissues) and excessive bleedings after a trauma. The severity of hemophilia A is directly related to the quantity of residual functional FVIII in the blood and can be severe (FVIII levels $<1 \%$ ), moderate (FVIII levels of $1-5 \%$ ) or mild (FVIII levels $>5 \%$ and $<40 \%$ ) (1). A limitation in this classification is the significant variability in the hemorrhagic phenotype observed in patients (2). Without the appropriate treatment, patients with hemophilia A have recurrent hemarthrosis, causing joint disease (arthropathy) and disability. There is a direct relationship between the number of hemarthrosis events and the condition of the joints, more than 2 hemorrhages in a joint could already show abnormalities in the radiography and physical examination (3).

Treatment is based on prophylactically or therapeutically replacing the deficient factor. Prophylactic administration to maintain FVIII trough levels $>1 \%$ [1 IU/dL of FVIII level] to change the patient's bleeding phenotype from severe to moderate has been the basis of prophylaxis over the years, it has been shown to be effective in preventing hemorrhages and thus the development of hemophilic arthropathy $(4,5,6)$. It is known that prophylaxis treatment based on fixed-dose, weight-based protocols or determined by bleeding patterns can be effective but does not protect all patients with hemophilia from joint damage. As with many drug treatments, there is a significant variability in the response among patients. For example, 2 studies conducted on adult and pediatric patients observed that the half-life of recombinant FVIII varied between 6 and 25 hours $(h)(7,8)$. Using pharmacokinetic data from 3 studies, it was observed that, for patients between 1 and 6 years of age (when comparing treatments with longer half-lives to those with shorter half-lives), the amount of time the FVIII plasmatic levels was above $1 \%$ after a standard infusion of $30 \mathrm{IU} /$ $\mathrm{kg}$ varied from $43 \mathrm{~h}$ to $77 \mathrm{~h}$, i.e., a difference of $34 \mathrm{~h}$. In older patients, the difference was $59 \mathrm{~h}$, ranging from $51 \mathrm{~h}$ to $110 \mathrm{~h}$ (9). Therefore, a wide difference in factor level among patients is to be expected, even after the infusion of equivalent doses. However, FVIII is generally prescribed based on the empirical fact that the average in vivo recovery is $2 \mathrm{IU} / \mathrm{dL}$ for each $1 \mathrm{IU} / \mathrm{kg}$ of infused factor VIII, and its half-life is approximately $12 \mathrm{~h}$. The amount of time an individual remains below a specific factor level is a determinant in the efficacy of prophylaxis. This will depend especially on the half-life of the infused factor, the administration rate, the in vivo recovery and the infused dose. A better understanding of the effect of the pharmacokinetics and administration rate of factor levels in patients in prophylaxis will help to better adapt the therapeutic regimens to patients and improve the costeffectiveness of clotting factor concentrates. The use of pharmacokinetics as a tool in treating hemophilia has been hindered by practical difficulties in implementing pharmacokinetic studies in patients to obtain data for dose adjustments. A study conducted according to the generally accepted directives first requires a washout period (prophylaxis not permitted for $72 \mathrm{~h}$ ) to allow any FVIII from previous treatments to decrease to baseline levels and then the collection of 9-10 blood samples for FVIII:C determination throughout the next 48 $\mathrm{h}$ following the infusion of the study dose. The dosing of conventional drugs (e.g., digoxin, aminoglycosides and immunosuppressants) based on plasma concentrations using limited blood sampling during regular treatment, i.e., therapeutic monitoring of drugs, has been performed for many years. The pharmacokinetic calculations of limited sampling (simplified PK) are often made through the Bayesian analysis. In this procedure, the values of the most likely PK parameters in each patient are calculated with the limited available data, based on previous knowledge of the drug's pharmacokinetics in the general patient population. In fact, FVIII dose optimization using the Bayesian analysis has been suggested in previous studies, however, it has not been systematically studied or evaluated in the context of prophylactic treatment for hemophilia (10).

If pharmacokinetics is to be used in standard clinical practice to personalize prophylaxis, a simplified measuring method is needed. Recent studies have evaluated the estimation of Bayesian pharmacokinetics based on pharmacokinetic models for patients with hemophilia $(10,11)$. This approach provides an estimation of an individual's FVIII pharmacokinetics based on 2 or 3 plasmatic samples to determine FVIII:C, with no need for a washout period. MyPKFiT®, a CE-marked medical device, is an online medical application designed to create an individual pharmacokinetics profile and determine the FVIII dosage regimen that would be appropriate for pharmacokinetics-guided prophylaxis in patients with hemophilia A. MyPKFiT ${ }^{\circledR}$ uses a population model published for Advate ${ }^{\circledR}$ (not validated for other FVIII products), along with a Bayesian algorithm to estimate the individual pharmacokinetic parameters. The patient information included in the system (age, weight, endogenous FVIII level) and 2-3 individual plasma FVIII levels help the device simulate and suggest various regimens to maintain the patient above a trough FVIII level for the specific interval of infusion selected based on the predicted individual pharmacokinetic profile. The dynamic interface allows the hematologist to share personalized pharmacokinetics-guided dosage options with the patient. There is broad consensus about the fact that the dosage for the prophylactic treatment of hemophilia should be individualized (12). The personalized prophylaxis enables treatment optimization, adapting it to the individual needs (pharmacokinetics, bleeding-phenotype, physical activity and joint condition) (13), which guaranties the right protection of the patient and the optimization of factor consumption.

To date, there are no actual data for the Spanish population that describe the interindividual 
pharmacokinetic differences that could increase the risk of hemorrhage or extend the time between infusions in patients with hemophilia A on prophylactic treatment. The aim of this observational, prospective multicenter study is to describe the pharmacokinetic profile of patients with hemophilia A in prophylaxis with Advate ${ }^{\circledR}$ in Spain using myPKFiT ${ }^{\circledR}$. As secondary objectives, we propose (1) the characterization of the educational role of pharmacokinetics for patients with hemophilia A undergoing prophylactic treatment in Spain, (2) identifying whether there are any changes in attitude among the patients after receiving the educational information on pharmacokinetics and (3) evaluating the role of pharmacokinetics and other individual factors in patients with hemophilia A undergoing prophylactic treatment in Spain.

\section{MATERIALS AND METHODS}

Study design: Observational, prospective (EPA-SP) and multicenter study with the participation of 14 centers from Spain.

Study population: The study will include patients with diagnosis of hemophilia A undergoing prophylactic treatment with Advate ${ }^{\circledR}$ who meet the following inclusion criteria:

- Male patients.

- Age between 1 and 65 years.

- Patients diagnosed with hemophilia A who are undergoing prophylactic treatment with Advate ${ }^{\circledR}$.

- Patient's endogenous FVIII levels $\leq 5 \%$.

- A signed informed consent form.

- Patients over 18 years old must have signed the informed consent form.

- In case of mature minors, their consent will also be obtained, along with their legal guardian's signed consent.

- For patients legally incapacitated to give their consent, the primary caregiver's consent, as patient's legal representative, will be obtained.

The patients must not meet any of the exclusion criteria:

- Withdrawal of informed consent.

- A medical or psychological condition that, in the investigator's opinion, impedes the patient from following the procedures of standard clinical practice.

- A concomitant diagnosis of other hemostasis disorders.

- Undergoing treatment for induction of immunologic tolerance at the time of inclusion.

The withdrawal criteria are defined as follows:

- Patients may revoke their consent for the use of their data at any time, without justifiying the decision. In no case will their medical care be affected. If a patient revokes his consent, his data will not be included in the analysis of results.

- Patients with FVIII-inhibitor antibody detected during follow-up.

Enrollment and follow-up period: The study will start in the last quarter of 2017 after obtaining the corresponding approvals from the regulatory agencies. The enrollment period will last 24 months. The follow-up period will be at least 12 months.

Visits definition: For the study, visits have been defined as follows:

- Historical: These are the data from the 12 months before starting to use myPKFiT ${ }^{\circledR}$ for adjusting the dose of PK-guided prophylaxis.

- Baseline: First day of using myPKFiT ${ }^{\circledR}$ for adjusting the dose of PK-guided prophylaxis.

- Inclusion visit: Day in which the patient is included in the study (signs the inform consent).

- 12 month follow-up visit (+ 1 month): It will be the visit after 12 months of the inclusion visit. It has a window of +1 month.

- 24 month follow-up visit (- 1 month): It will be an optional visit 24 months after the inclusion visit. It has a window of - 1 month.

Treatment description: The study will assess the pharmacokinetics of Advate ${ }^{\circledR}$, which contains octocog alpha [antihemophilic factor (recombinant), plasma/ albumin-free method, rAHF-PFM"], a purified protein with 2332 amino acids. The treatment is produced by recombinant DNA technology carried out in Chinese hamster ovary cells. The product is prepared with no addition of any protein (exogenous) of human or animal origin in the cell culture, purification or final formulation processes.

Sample size: Given that this will be an observational study, all patients who meet the inclusion criteria and none of the exclusion criteria will be prospectively included. The study will also include a cohort of patients who are already using myPKFiT ${ }^{\circledR}$ for the PK-guided prophylaxis dose adjustment. The number of patients predicted for enrollment in the study period will be at least 100 .

Data source: Data will be obtained from the patient's medical history and interview. The results of the pharmacokinetic analysis will also be obtained. If the patient is already using myPKFiT ${ }^{\circledR}$ at the start of this study, all pharmacokinetics data will be retrospectively collected. We will also record historical data: weight, physical activity, joint condition, total number of hemorrhagic episodes, total number of hemarthrosis episodes, treatment regimen and annual factor consumption. All of these data will be taken from the year prior to the start of the pharmacokinetics-adjusted prophylaxis using myPKFiT ${ }^{\circledR}$, if they are available. Assessment variables: demographic variables, clinical variables, personal history, disease characterization variables, analytical variables, treatment variables, educational role characterization variables and safety variables will be recorded (Table 1).

Case selection: All patients with a diagnosis of hemophilia A undergoing prophylactic treatment with Advate $^{\circledR}$ under follow-up by the recruiting centers will be offered to participate in this study. The study 
will also include a cohort of patients who are already using myPKFiT ${ }^{\circledR}$ for the PK-guided prophylaxis dose adjustment.

Data collection: Investigators will record the patient's interview data and medical history, with demographic information, personal history especially regarding hemophilia, as well as information on physical activity. These data will be collected retrospectively on the day the patient is included in the study and will contain the information of the 12 previous months. This information will also be collected on each successive visit.

To evaluate the adherence to the treatment, the VERITASPro questionnaire (14) will be used. This is a validated questionnaire to assess self-reported adherence of prophylactic treatment in patients with hemophilia A. It will be evaluated only in patients that will start using myPKFiT $^{\circledR}$ after the inclusion in the study.

For all patients, 2 samples will be taken on the day of inclusion according to criteria established in the myPKFiT ${ }^{\circledR}$ user manual ${ }^{\circledR}$ v2.0. The first sample will be taken between 3-4 h ( \pm 30 minutes) after completing the Advate ${ }^{\circledR}$ infusion, and the second sample will be taken 24$32 \mathrm{~h}$ ( \pm 60 minutes) after the infusion.

For patients already using myPKFiT ${ }^{\circledR}$ before the start of the study, pharmacokinetics data will be collected from baseline day, that is the day when the patient began dose adjustment using myPKFiT ${ }^{\circledR}$. This information will be collected retrospectively.

To assess whether the pharmacokinetics-guided prophylaxis using myPKFiT ${ }^{\circledR}$ in the Spanish population can be performed correctly using both 1-stage clotting method and chromogenic method, we will analyze the FVIII activity of one of the samples collected during the study (preferably inclusion day one) from patients recruited by La Paz University Hospital, Vall D'Hebron University Hospital and Virgen del Rocío University Hospital (the estimate number will be approximately 50 patients). The samples from these patients will be analyzed by both methods in all 3 hospitals, which will help reduce the intrahospital and interhospital bias.

Electronic CRF: An electronic CRF (Xolomon ${ }^{\circledR}$ ) that will contain the variables specified in Table 1 will be designed. Study Timeline Table 2 details the study timeline.

Table 2. Study Timeline

\begin{tabular}{|c|c|c|c|c|c|c|}
\hline & 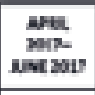 & mong & 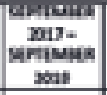 & gentuana & 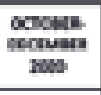 & 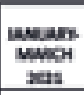 \\
\hline 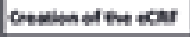 & * & & & & & \\
\hline 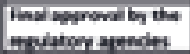 & & - & & & & \\
\hline 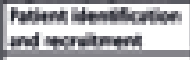 & & & $*$ & & & \\
\hline Data ecolection & & & $*$ & $*$ & & \\
\hline Nebink tobsorup & & & - & $\cdot$ & & \\
\hline 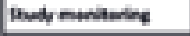 & & & * & * & & \\
\hline batibunt colume & & & & $*$ & & \\
\hline Ratatical andins & & & & & - & \\
\hline 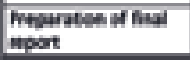 & & & & & & - \\
\hline
\end{tabular}

Statistical analysis of the results: Categorical variables will be expressed as frequency and percentages. The quantitative variables as mean, median, standard deviation and confidence interval with a confidence level of $95 \%$. The contrast of the distribution between categorical variables and the differences between groups of quantitative variables will be performed using the statistical chi-squared test or Student's t-test if they are parametric. If the distribution is not normal, the nonparametric tests will be used.

Analyses will be performed as well of subgroups for variables of importance, such as PK parameters, age, hemorrhagic phenotype, patients with an inhibitor history, physical activity or financial impact.

All these analyses will be performed using the statistical environment R (R Core Team [2013]. R: A language and environment for statistical computing; R Foundation for Statistical Computing, Vienna, Austria) with RStudio (RStudio Team [2015], RStudio: Integrated Development for R. RStudio, Inc., Boston, MA).

Monitoring the quality of the study: The study will be followed up by monitors from the Central Unit of Clinical Research and Clinical Trials (UCICEC) of University Hospital La Paz - IdiPAZ. Online monitoring and other in-person monitoring will be performed at regular intervals, as well as telephone calls to the investigators. During the visits, the monitor will review the patients' original records and will assess the procedures to ensure the study's quality.

Communication of adverse events: The investigators will report all adverse events to the health authorities and to Baxalta Laboratory "now part of Shire".

Ethical issues: The researchers will strictly adhere to the provisions of this protocol and the standards of good clinical practice. Following the directives on observational studies, the study has been approved by the Drug Research Ethics Committee of La Paz University Hospital. Only the researchers will know the data (initials and history number) that could identify the patient. The patient's initials and history number will be separated from the rest of the notebook and will not be entered into the study database. The patient will be identified with a numerical code to respect personal data confidentiality, according to Organic Law 15/1999 on the protection of personal data. The collection and handling of samples will follow the indications established in Law 14/2007 of July 3 on biomedical research.

A manuscript will be prepared in a maximum term of 6 months after closure of the study database for its publication in a journal of that specialty. The overall data will be used in publications for medical congresses or journals, which will reference the study and the research group. This study has been recorded in the Clinicaltrials.gov database with the code NCT03006965.

\section{DISCUSSION}

The most common hemorrhagic manifestations of hemophilia are recurrent hemarthrosis, which lead to progressive joint destruction. Thus, preventing arthropathy is one of the main objectives of hemophilia treatment. 
Prophylactic treatment has been shown to slow the progression of arthropathy but not to prevent it. There are studies that have demonstrated the superiority of prophylactic treatment versus on-demand treatment in preventing joint injury $(5,6)$.

Treatment with FVIII, as with many other drugs, presents significant interindividual variability. Pharmacokinetics can explain a large part of this variability. Understanding pharmacokinetics will therefore help personalize the treatment, as will taking into account personal factors such as hemorrhagic phenotype and physical activity. We also consider that the patient's understanding of the importance of the pharmacokinetic parameters helps improve therapeutic adherence.

The use of simple tools that help estimate the pharmacokinetic parameters based on the Bayesian method, such as myPKFiT ${ }^{\circledR}$, can contribute to making its use easy for patients.

This study will allow us to obtain FVIII pharmacokinetic data from the Spanish population, which in the future will allow us to enrich ourselves and obtain greater precision in dose adjustment for this patient population.

\section{TRIAL INVESTIGATION GROUP}

Spanish Society of Thrombosis and Haemostasis prophylaxis group:

Alonso-Saladrigues A, Bernardo-Gutiérrez A, Butta-Coll N, Campano-Morillo C, Caunedo-Almagro P, Cerezo- Manchado JJ, Cid-Haro AR, Costa-Pinto J, de la Corte- Rodríguez H, Fernández I, Fernández-Docampo M, Gago-Caballero B, Galmes Sureda B, García-Barcenilla S, Martín-Salces M, Martínez-Carballeira D, Martorell Murtra M, Megias-Vericat JE, Palomero-Massanet A, Parra-López R, Pérez-Alenda S, PérezRodríguez A, Querol-Fuentes F, Rivas-Pollmar I, Sánchez Raga JM, Sierra-Aisa C.

\section{ACKNOWLEDGEMENT}

This work has had the support of the Spanish Clinical Research Network (SCReN), funded by ISCIII-Subdirección General de Evaluación y Fomento de la Investigación (project PT13/0002/0015), integrated in Plan Estatal de I+D+I 2013-2016 , and co-financed by Fondo Europeo de Desarrollo Regional (FEDER)

\section{REFERENCES}

[1]. Blanchette VS, Key NS, Ljung LR, Manco-Johnson MJ, Van Den Berg HM and Srivastava A For the Subcommittee on Factor VIII, Factor IX and Rare Coagulation Disorders. Definitions in hemophilia: communication from the SSC of the ISTH. Journal of Thrombosis and Haemostasis 2014; 12 (11): 19351939.

[2]. Pavlova A, Oldenburg J. Defining Severity of Hemophilia: More than Factor Levels. Semin Thromb Hemost 2013; 39 (7): 702-710.

[3]. Funk MB, Schmidt H, Becker S, Escuriola C, Klarmann D, Klingebiel T, et al. Modified magnetic resonance imaging score compared with orthopaedic and radiological scores for the evaluation of haemophilic arthropathy. Haemophilia 2002 Mar; 8 (2): 98-103.
[4]. Astermark J, Petrini P, Tengborn L, Schulman S, Ljung R, Berntorp E. Primary prophylaxis in severe haemophilia should be started at an early age but can be individualized. Br J Haematol 1999; 105 (4): 1109-13.

[5]. Manco-Johnson MJ, Abshire TC, Shapiro AD, Riske B, Hacker MR, Kilcoyne R, et al. Prophylaxis versus episodic treatment to prevent joint disease in boys with severe hemophilia. N Engl J Med 2007; 357 (6): $535-44$.

[6]. Gringeri A, Lundin B, von MS, Mantovani L, Mannucci PM. A randomized clinical trial of prophylaxis in children with hemophilia A (the ESPRIT Study). J Thromb Haemost 2011; 9 (4): 700-10.

[7]. Bjorkman S, Folkesson A, Jonsson S.

Pharmacokinetics and dose requirements of factor VIII over the age range of 3-74 years: a population analysis based on 50 patients with long-term prophylactic treatment for haemophilia A. Eur J Clin Pharmacol 2009; 65: 989-98.

[8]. Bjorkman S, Blanchette VS, Fischer K, Oh M, Spotts G, Schroth P, et al. Comparative pharmacokinetics of plasma and albumin-free recombinant factor VIII in children and adults: the influence of blood sampling schedule on observed agerelated differences and implications for dose tailoring. J Thromb Haemost 2010; 8: 730-6.

[9]. Collins PW, Fischer K, Morfini M, Blanchette VS, Björkman S; International Prophylaxis Study Group Pharmacokinetics Expert Working Group. Implications of coagulation factor VIII and IX pharmacokinetics in the prophylactic treatment of haemophilia. Haemophilia 2011; 17 (1): 2-10.

[10]. Bjorkman S. Limited blood sampling for pharmacokinetic dose tailoring of FVIII in the prophylactic treatment of haemophilia A. Haemophilia 2010, 16 (4): 597-605.

[11]. Bjorkman S, Oh M, Spotts G, Schroth P, Fritsch S, Ewenstein BM, et al. Population pharmacokinetics of recombinant factor VIII: the relationships of pharmacokinetics to age and body weight. Blood 2012 Jan 12; 119 (2): 612-8.

[12]. Srivastava A, Brewer AK, Mauser-Bunschoten EP, Key NS, Kitchen S, Llinas A, et al. Treatment Guidelines Working Group on Behalf of The World Federation Of Hemophilia. Guidelines for the management of hemophilia. Haemophilia. 2013; 19 (1): e1-47.

[13]. Valentino LA. Considerations in individualizing prophylaxis in patients with haemophilia A. Haemophilia. 2014; 2(5): 607-15.

[14]. Duncan N, Kronenberger W, Roberson C, Shapiro A. VERITAS-Pro: a new measure of adherence to prophylactic regimens in haemophilia. Haemophilia 2010 Mar; 16(2): 247-55.

[15]. Hemophilia Joint Health Status Score from World Federation of Hemophilia. Link: http://www.wfh.org/ en/page.aspx?pid=885. 
Table 1.

\begin{tabular}{|c|c|c|c|c|c|}
\hline Variatble & Hiheerical' (1 year) & Masultae? & $\begin{array}{l}\text { Indenios } \\
\text { vidin' }\end{array}$ & 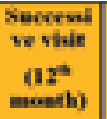 & $\begin{array}{l}\text { Seccersive } \\
\text { visit } \\
\text { (24) } \\
\text { nomih) }\end{array}$ \\
\hline \multicolumn{6}{|l|}{ - Dereographic } \\
\hline * Aev, Height & & $x^{4}$ & $\mathrm{x}$ & $\mathbf{x}^{2}$ & $\overline{\mathbf{x}^{4}}$ \\
\hline * Weighn & $\mathbf{x}$ & $\mathbf{x}$ & $\mathbf{x}$ & & $\mathbf{x}$ \\
\hline \multicolumn{6}{|l|}{ Chaical } \\
\hline - Date of dagnonis of hemophiala A & & & $x$ & & \\
\hline - Severity of hemophilis A (uevere, moderste, mild) & & & $\bar{x}$ & & \\
\hline - Trpe of Fil gene mutation & & & $\mathrm{x}$ & & \\
\hline - Starting date of prophylesils & & & $\mathrm{x}$ & & \\
\hline 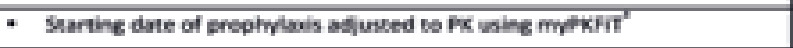 & & & $\bar{x}$ & & \\
\hline 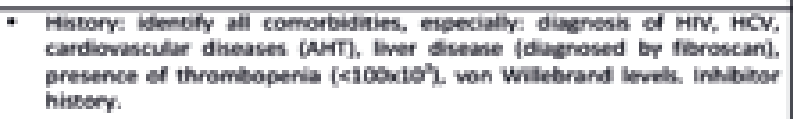 & & & $\mathbf{x}$ & & \\
\hline 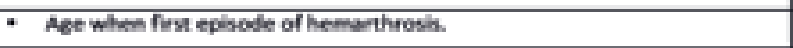 & & & $\mathrm{x}$ & & \\
\hline 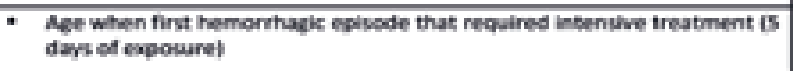 & & & $\mathbf{x}$ & & \\
\hline \multicolumn{6}{|l|}{ Characterikation of the diverave } \\
\hline - Severity of the prewlous hemonthaeeh, according to the Eurosean & $\mathbf{X}$ & $\mathbf{x}$ & $\bar{x}$ & $\bar{x}$ & $\bar{x}$ \\
\hline 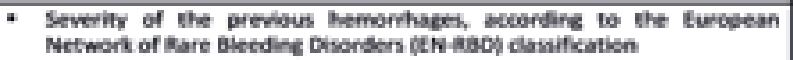 & $\mathbf{x}$ & $\mathbf{x}$ & $\mathbf{x}$ & $\mathbf{x}$ & $\mathbf{x}$ \\
\hline * Number of hoiphaluations for hemophita in the part year & $\mathbf{x}$ & $\bar{x}$ & $\bar{x}$ & $\mathbf{x}$ & $\mathbf{x}$ \\
\hline - Hemonrhagic risk of physical activity according to the Anoderick scale & $\mathbf{x}$ & $\bar{x}$ & $\mathrm{x}$ & $\mathbf{x}$ & $\mathbf{x}$ \\
\hline 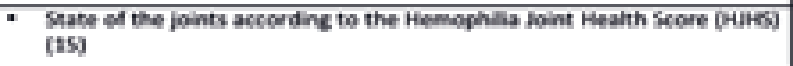 & $\mathbf{x}$ & $\mathbf{x}$ & $\mathrm{x}$ & $\mathbf{x}$ & $\mathbf{x}$ \\
\hline 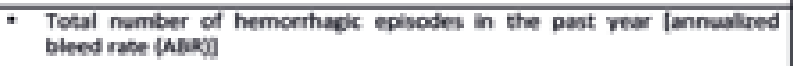 & $\mathbf{x}$ & $\mathrm{x}$ & $\mathbf{x}$ & $\mathbf{x}$ & $\mathbf{x}$ \\
\hline * Total number of hemarthrous events in ENe past vear (AabK)- & $\mathbf{x}$ & $\bar{x}$ & $\mathrm{x}$ & $\bar{x}$ & $\bar{x}$ \\
\hline * Concomitust medicatien & $\mathbf{x}$ & $\bar{x}$ & $\bar{x}$ & $\bar{x}$ & $\bar{x}$ \\
\hline 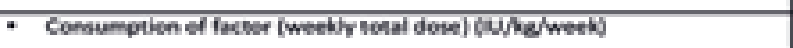 & $\mathbf{x}$ & $\mathrm{x}$ & $\mathrm{X}$ & $\bar{x}$ & $\bar{x}$ \\
\hline \multicolumn{6}{|l|}{ - Latorabary bets } \\
\hline 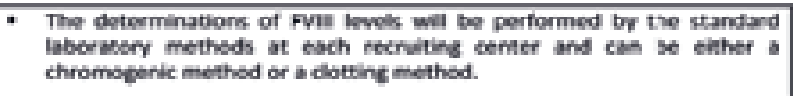 & $\mathbf{x}$ & $\mathbf{x}$ & $\mathbf{x}$ & $\mathbf{x}$ & $\mathbf{x}$ \\
\hline 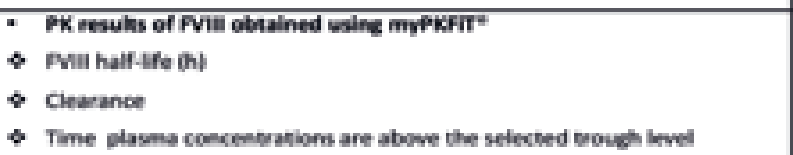 & & $\mathbf{x}$ & $\mathbf{x}$ & $\mathrm{x}$ & $\mathbf{x}$ \\
\hline \multicolumn{6}{|l|}{ Treatment } \\
\hline 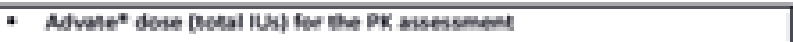 & $\bar{x}$ & $\bar{x}$ & $\bar{x}$ & $\bar{x}$ & $\bar{x}$ \\
\hline 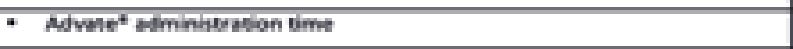 & $\mathbf{X}$ & $\bar{x}$ & $\bar{x}$ & $\bar{x}$ & $\bar{x}$ \\
\hline - Selected infuilon rate & $\mathbf{X}$ & $\bar{X}$ & $\bar{x}$ & $\bar{x}$ & $\bar{x}$ \\
\hline - Safeg & & & & & \\
\hline - Record of adverse events & & & $\mathrm{x}$ & $\bar{x}$ & $\bar{x}$ \\
\hline - Adbereace to treatricat & & & & & \\
\hline 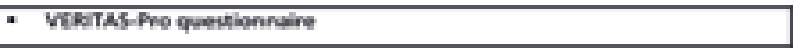 & & & $\mathrm{x}^{5}$ & $x^{2}$ & $x^{9}$ \\
\hline * Characterienation of the edecational rele & & & & & \\
\hline - Nurve's role in the manacememt of hemophiala & & & $\bar{x}$ & $\bar{x}$ & $\bar{x}$ \\
\hline
\end{tabular}

1. Data from 1 year previous to the beginning of using myPKFiT ${ }^{\oplus}$ for the PK-guided prophylaxis dose adjustment.

2. Baseline visit: it is the visit which the patient began using myPKFiT ${ }^{\oplus}$ for the PK-guided prophylaxis dose adjustment. Only valid for the cohort of patients who were

already using myPKFiT ${ }^{\oplus}$ at the time of inclusion in the study.

3. Inclusion visit: it is the day on which the patient signs the inform consent.

4. Only valid for the cohort of patients who started using myPKFiT ${ }^{\oplus}$ for the PK-guided prophylaxis dose adjustment at the time of inclusion in the study. 